\title{
Simulation of Company's Bankruptcy Probability based on Catastrophe Theory
}

\author{
Ramzan E. Janshanlo ${ }^{1 *}$, Miras E. Noyanov ${ }^{1}$ and Gulsagat T. Andybayeva ${ }^{2}$ \\ 'Al-Farabi Kazakh National University, Almaty, 050040, Republic of Kazakhstan; \\ ramazan1951@mail.ru, caercauthor@yahoo.com \\ ${ }^{2}$ Narxoz University, Zhandosova, Almaty, 050035, Republic of Kazakhstan;
} caercauthor@yahoo.com

\begin{abstract}
Objectives: This research addresses the issues of construction of mathematical models to estimate the company's bankruptcy probability. The aim of the paper is to reveal object behaviour in different situations, all the possible interrelations, principles and conditions of development should be taken into consideration with the models. Methods/Statistical Analysis: The authors suggest the methodology to measure the bankruptcy probability based on the catastrophe theory concepts. The historical analogy approach, methods of comparison study, the catastrophe theory and the multiple correlative-regressive analyses were used as a methodological framework for the research. Findings: In the article, there are findings from the analysis of basic forecasting models for the company's bankruptcy probability, their main advantages and disadvantages are given too. As a result, the mechanism for a company development simulation using the catastrophe theory was developed. Applications/Improvements: This helps to avoid a crisis and a financial default of a company when a definite forecasting model is made at the appropriate time. The company's bankruptcy probability analysis can be considered a basic method giving an opportunity to plan future economic status of a company.
\end{abstract}

Keywords: Bankruptcy, Catastrophe Theory, Financial Stability, Recession, Risks

\section{Introduction}

Financial and operational activities are associated with many entrepreneurial risks. The risks influence became more evident with a recession. Financial risks are the most important among the others because they put further successful operation of the company at threat. Their influence leads to a decrease in financial performance and a financial strength of a company and, finally, to bankruptcy. Bankruptcy is a widely spread problem that is why it is vitally to determine adverse trends early. Bankruptcy probability is one of the evaluating characteristics for the current financial status of the company. Company management is able to keep the probability low if they make the analysis from time to time and use reasonable efforts at the proper time.

Academic literature suggests different methods and mathematical models for companies' crisis and bankruptcy diagnostics. Companies from the developing economics use the models for bankruptcy risk diagnostics by ${ }^{1-7}$ etc. made with the apparatus of the multivariate discriminant analysis and giving an opportunity to an approximate division into two groups of companies: Potential bankrupts and financially strong companies.

In the developing economies, there are their own decisions, which take into account economic particularities. As an example, we can take models developed by the Russian scientists, such as ${ }^{-12}$ etc. In their papers, criteria and methods to estimate the company's bankruptcy probability are considered in depth, as well as there is a review of quantitative and qualitative aspects influencing the company's financial stability.

Unlike the classical methods of the company's bankruptcy probability analysis, the catastrophe theory is a universal tool to explore drastic, intermittent transitions, sudden qualitative changes in a non-linear dynamic

* Author for correspondence 
system state when their parameters are being changed, moreover, the status dependence on some separate parameters and their part is presented in an explicit form.

In 同 $^{2}$ were founders of the catastrophe theory. A great contribution in the theory development was also made by ${ }^{15-19}$ etc. As Arnold mentioned, 'the catastrophe theory gives a universal method to study all intermittent transitions, disruptions, qualitative changes ${ }^{14}$. At the same time, the number of applications for the catastrophe theory is very limited.

As the loss of financial stability in the company goes under an influence of basic development mechanisms, the conclusions of the theory absolutely suit the analysis of the conditions when the company stops developing sequentially and enters a bifurcation phase. Considered from this perspective, conclusions of the catastrophe theory are essential for the analysis of conditions helping to avoid unwanted bifurcations and the company's bankruptcy.

\section{Methodology}

\subsection{Traditional Approach}

In modern economic literature, there are many approaches to the analysis and estimation of the company's bankruptcy probability ${ }^{1-7}$. To assess the company's bankruptcy probability, foreign companies often use Altman Z-Score (credibility index), which is a five-factor model, made with a use of data on successful and bankrupt manufacturing enterprises from the USA ${ }^{1}$. The method was proposed in 1968 by Altman. The credibility index was designed using the apparatus of the multivariate discriminant analysis and it allows at a first approximation dividing economic entities into potential bankrupts and non-bankrupts.

For the index design, Altman reviewed 66 companies, a half of which had done bankrupt in 1946-1965, while the others had been successful. He also examined 22 analytical coefficients that could be useful to predict possible bankruptcy. From these indicators, he selected five most significant ones and made a multiple regression equation. Thus, the Altman index is a function of several parameters that describe an economic strength of a company and results of its activities for the time elapsed.

The resulting coefficient of the bankruptcy probability Z-Score is calculated with five factors and in general, the credibility factor looks like:
Z-Score $=1.2 \mathrm{~K}_{1}+1.4 \mathrm{~K}_{2}+3.3 \mathrm{~K}_{3}+0.6 \mathrm{~K}_{4}+\mathrm{K}_{5}$,

Where $\mathrm{K}_{1}$ is circulating capital share in the active assets; $\mathrm{K}_{2}$ is ratio of the retained profits to the assets; $\mathrm{K}_{3}$ is ROA; $\mathrm{K}_{4}$ is ratio of market price of the company's whole equity and privilege shares to the debt funds; $\mathrm{K}_{5}$ is asset turnover.

Depending on the Z-Score value, the bankruptcy probability is measured for a two-year period using the definite scale: if $Z<1.81$, the bankruptcy probability is very high; if $1.81<Z<2.675$, the bankruptcy probability is medium; if $Z=2.675$, the bankruptcy probability is 0.5 ; if $2.675<\mathrm{Z}<2.99$, the bankruptcy probability is not high; if $Z>2.99$, the bankruptcy probability is insignificant.

The result index, Altman $\mathrm{Z}$ score, is a result of the discriminant function. Obviously, with a higher value of $\mathrm{Z}$, companies are healthier. Altman found that companies with their Z-score over 2.99 would demonstrate financial stability and no further complications in their business. Companies with Z-score under 1.81 (of course, including negative numbers), would eventually reach bankruptcy. Only a small group of such companies would survive. For companies with Z-score 1.81-2.99, it was impossible to make a clear and convincing forecastit.

At the same time, the Z-score has a serious deficiency as it can be used essentially only for large-scale companies that quote their shares on stock exchanges. It is for these companies that we can obtain an objective market assessment of their own capital.

Altman got a modified version of his formula for companies, shares of which had not been quoted on a stock exchange:

$\mathrm{R}=8.38 \mathrm{X}_{1}+\mathrm{X}_{2}+0.054 \mathrm{X}_{3}+0.63 \mathrm{X}_{4}$,

Where $\mathrm{X}_{4}$ is a book value instead of a market value of shares.

The Altman index is among the most common. However, a careful study of it shows that it was incorrectly made: $\mathrm{X}_{1}$ is associated with crisis management, $\mathrm{X}_{4}$ describes an onset of a financial crisis, while the others describe the onset of an economic one. From the system perspective, this indicator has no right to exist.

An advantage of tools similar to the Altman's model is a high probability with which they predict bankruptcy about two years before an actual notice of a tender, while their disadvantage is less statistical reliability of results in case of forecasts for the distant future. 
Table 1. System of Beaver indice to detect bankruptcy

\begin{tabular}{lccc}
\hline Indice & \multicolumn{3}{c}{ Indice values } \\
\cline { 2 - 4 } & Favourable & $\begin{array}{c}5 \text { yrs before } \\
\text { bankruptcy }\end{array}$ & $\begin{array}{c}\text { 1 yr before } \\
\text { bankruptcy }\end{array}$ \\
\hline Beaver index & $0.40-0.45$ & 0.17 & -0.15 \\
Return on assets, \% & $6-8$ & 4 & -22 \\
Leverage ratio, \% & $<37$ & $<50$ & $<80$ \\
Short-term assets cover ratio & 0.40 & $<0.30$ & $<0.06$ \\
with own working capital & & & $<1$ \\
Current ratio & $<3.2$ & $<2$ & $<$ \\
\hline
\end{tabular}

Beaver was the first financial analyst to use statistical methods together with financial ratios to predict probable bankruptcy of a company. He proposed five-factor model to assess a financial state of a company to detect bankruptcy and it contains the following indicators Table $1^{2}$ :

\section{Return on assets;}

Proportion of borrowed funds in liabilities;

Current ratio;

Proportion of net working capital in assets;

Beaver index $=($ net profit + depreciation $) /$ borrowed funds.

The analyst studied problems of companies associated with an inability to meet their financial obligations (or difficulties with repayment of a debt as per schedule). Beaver made a database, which used later in his model for statistical testing of reliability against 30 financial ratios. Average values of these indicators from companies without financial difficulties were compared with values of indicators from companies that later went bankrupt. Assessment criteria for the Beaver index: $\mathrm{N} \leq 0.17$ high bankruptcy probability; $0.17<2 \mathrm{~N} \leq 0.4$ - medium bankruptcy probability; $\mathrm{N}>0.4$ - low bankruptcy probability.

Lis designed a discriminant model for the UK enterprises. The Lis's model to forecast insolvency (bankruptcy) of companies looks like:

$\mathrm{Z}=0.063 \mathrm{X}_{1}+0.092 \mathrm{X}_{2}+0.057 \mathrm{X}_{3}+0.001 \mathrm{X}_{4}$

Where $\mathrm{X}_{1}$ - working capital/total assets; $\mathrm{X}_{2}$ - profits from sales/total assets; $\mathrm{X}_{3}$ - retained earnings/total assets; $\mathrm{X}_{4}$ - equity/debt capital. Assessment criteria: $\mathrm{Z}<0.037$ - high bankruptcy probability; Z > 0.037 - medium bankruptcy probability.

It should be taken into account that for Kazakhstan companies the Lis's model to detect bankruptcy probability shows overestimates to some extent, as the profit on sales has a significant impact on a final score excluding financial activities and tax regime.

Taffler and Tisshaw proposed a four-factor model to predict insolvency (bankruptcy) based on the following approach. With computer equipment, in the first stage, they calculate 80 ratios using data on failed and solvent companies. Then, with the statistical method, known as a multivariate discriminant analysis, it is possible to make a model of solvency, identifying particular ratios with which we best distinguish two groups of companies and their coefficients. Such a selective count of ratios is typical to define some key measurements of a company's activities, a financial risk and liquidity. Combining these indicators and bringing them together in an appropriate way, the solvency model gives an accurate picture of a company's financial state. The Taffler's model looks like ${ }^{4}$ :

$Z=0.53 X_{1}+0.13 X_{2}+0.18 X_{3}+0.16 X_{4^{\prime}}$

Where $\mathrm{X}_{1}$ - profit from the sale/short-term liabilities; $\mathrm{X}_{2}$ - short-term assets/total liabilities; $\mathrm{X}_{3}$ - current liabilities/total assets; $\mathrm{X}_{4}$ - revenues from sales/total assets. Assessment criteria: $\mathrm{Z}>0.3$ - low bankruptcy probability, $\mathrm{Z}<0.2$ - high bankruptcy probability.

Leverage, profitability, liquidity, capital adequacy and other parameters were assessed in time of the Taffler's model design. Taken together, coefficients within the models give an objective picture of a risk of future insolvency and current solvency. However, these models have a number of disadvantages. First, they are focused on smooth changes in the system's state, excluding jump processes. Second, for example, models by Altman, Beaver, Lis, Taffler, etc. contain weighting and thresholds values for complex and particular indicators calculated on the basis of US and British analytics from 1960-1970-ies and do not meet circumstances of economic development 
in the developing countries with the CIS as an example. A comparison of data obtained for a number of countries shows that weighting and thresholds intervals do not only greatly vary from country to country and from year to year within the same country. It turns out that these models are not resistant to variations in input data.

\subsection{Catastrophe Theory}

The Catastrophe Theory as a branch in mathematics appeared as early as in the middle of the 20th century from the singularity of smooth mapping theory proposed by mathematician Whitney and the dynamic system bifurcation theory by 17,20 . It is a research program to study and forecast various systems' instability as the stability loss may lead to a catastrophe, results of which can differ greatly: From a start to a new development volute to a system death.

The theory supposes that the studied process is described with a certain number of control and internal parameters and it is possible to find out and predict a catastrophe of the system, modelling functions that consider relations between variables, which describe its behaviour. The evolutionary process of the system is mathematically described and a relation between variables has the form of a regression equation, the nature of which depends on the value of parameter $x$. If $x$ parameter is positive, but there is a tendency towards its decrease, the system is considered close to the catastrophe. The catastrophe is a stepwise change of the system in the form of a sudden system response to smooth changes in external conditions.

Issues of the reviewed company' financial stability by means of the catastrophe theory was considered by ${ }^{21-20}$, etc. However, these papers do not throw enough light on the application mechanism for the catastrophe theory.

The paper suggests an approach to explore the financial and economic crisis of companies, using the canonical fold and cusp catastrophes, mathematical models of company's crisis development at different levels of detail: 1. When assessing liquidity and solvency, as well as financial stability, economic activity and profitability; 2. When evaluating the general financial position of a company and 3. To model the developmental potential of a company.

The key method to study stepwise changes from a stable to an unstable state in a credit institution's activity is to find out if the real function has critical points, in which the derivative is equal to zero. Parameters of the objective function of the commercial bank's financial stability $F(x$, $Q, t)$ are also functions: $x=f\left(x_{1}, x_{2}, \ldots, x_{k}\right)$ - generalized development factor, where $x_{1}$ is technologies, $x_{2}$ is capital, $x_{3}$ is staff, etc.; $Q=f\left(c_{1}, c_{2}, \ldots, c_{k}\right)$ - bank financial position, where $c_{i}$ is various financial parameters; $\mathrm{t}$ is time being a linear parameter.

To identify a crisis at a credit institution, the Whitney's canonical cusp catastrophe was used:

$V(x, Q, t)=1 / 4 x^{4}+1 / 2 Q x^{2}+t x$,

Where $V(x, Q, t)$ is financial stability potential; $x$ is generalized development factor, which includes technologies, capital, HR, etc.; $Q$ is commercial bank's financial position; $t$ - time.

Strange as it may seem, successive works 4 4,25 have the same focus. That was the last suggestion concerning the assessment of the company's bankruptcy probability. So, the given problem has almost had no solutions.

\section{Materials and Methods}

\subsection{Algorithm to Review Bankruptcy Probability}

Any company is a complex economic system that cannot be in equilibrium for long as it is influenced by a number of factors: Its market position, dependence on external creditors and investors, competitors' pressure, efficiency of economic and financial operations, etc. That is why non-equilibrium states (or oscillations) can occur and the system can become unstable. Sudden changes in the complex system in the course of time can be hardly analyzed with classical mathematical methods. The catastrophe theory is preferable in this case. The theory establishes specific regularities associated with a system's sharp and sudden transition from one state to another, as well as with mechanisms of mode changes in the system dynamics. It also defines causes that lead to a discontinuity of changes in individual parameters and the system as a whole, make them jump to another level and pass to another qualitative state.

Catastrophes are stepwise changes in the system resulting from a gradual change in external conditions. The catastrophe theory studies sudden and sharp qualitative changes in the state of dynamical systems, resulting from slow, smooth, small changes in parameters 
The theory's approaches build up the methodology for modelling discontinuities in developing processes, allowing us to describe a variety of discontinuities from the mathematical point of view and obtain a satisfactory theoretical generalization for these phenomena. The catastrophe theory methods can be used for qualitative characteristics of various types of the economic dynamics by identifying geometric structures that reflect slow changes and peculiarities that occur in dynamical systems, described by smooth functions with a limited number of

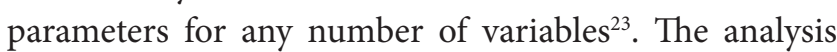
model for the company's bankruptcy probability based on the catastrophe theory is given in Figure 1.
The first stage includes a retrospective analysis of a company's activity and making a set of analytical indicators, which will be further used to assess the financial stability of the company. The second stage includes a choice of the most appropriate elementary catastrophe for the specific company based on hierarchically used canonical catastrophes. The third stage includes building models of the company's stable and unstable activities with the further calculated bankruptcy threat in the future.

The assessment of the company's financial stability involves the use of indicators, which provide the most accurate information on its level, direction and trend of changes. At the same time, economics still has not stated

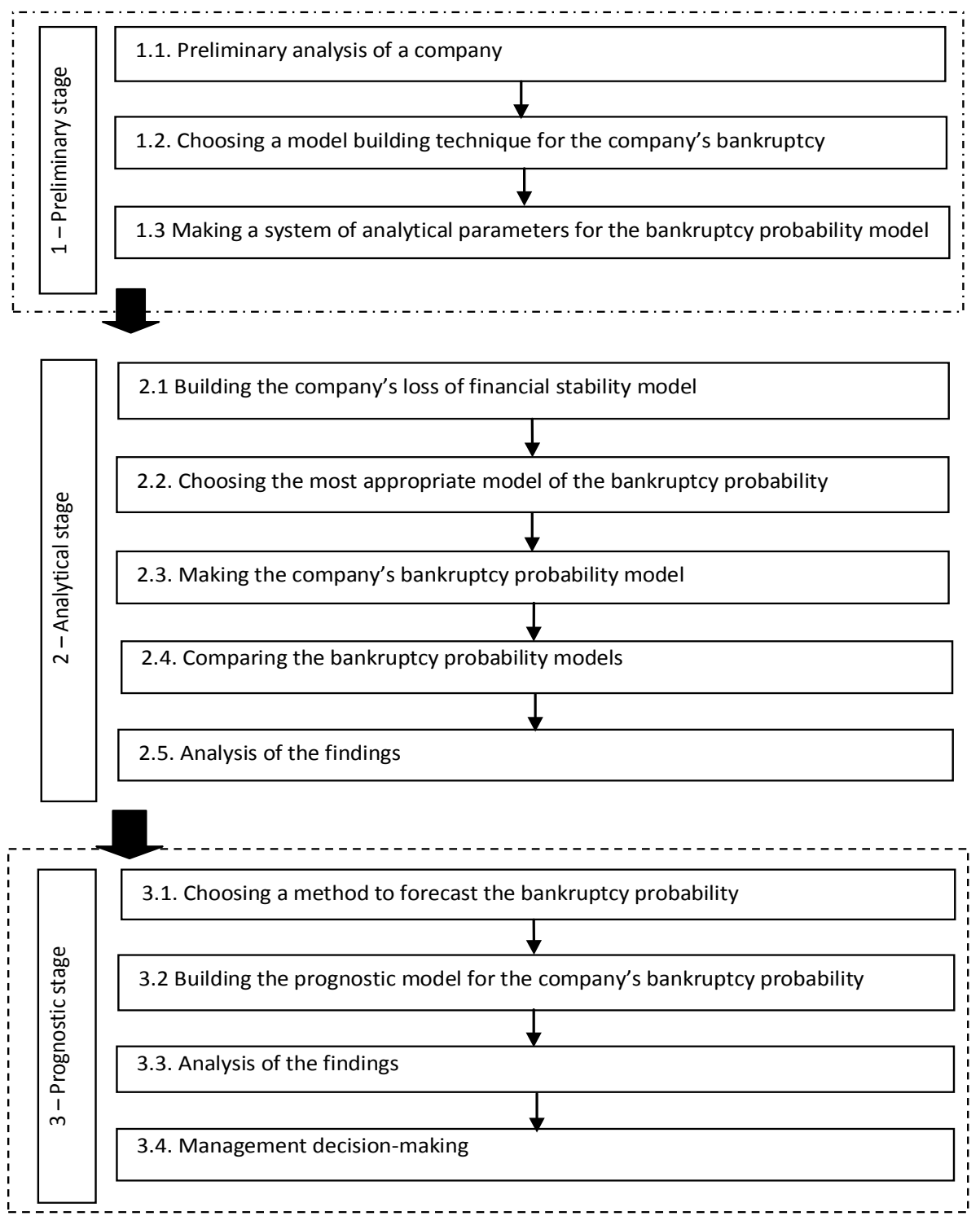

Figure 1. Stages of the company's bankruptcy probability analysis. Note: Made on the basis of ${ }^{25,26}$. 
approaches to indicators selection rationale, methods for their calculation and the definition of change tendencies. With absent generally accepted assessment standards, we have used the author's approach in the context of this research.

\subsection{Integral Assessment of Financial Stability}

The financial stability of a company is a stable financial position when the company has enough financial resources to perform its continuous activities. The usage of a set of parameters for an analysis of the financial stability is widespread in the economic analysis, though this method precludes any possibility to make an overall assessment. To get an overall idea of the company's financial stability, one must use an integrated parameter. The economic literature does not have any common methodology to develop the integrated parameter for the financial stability. The problem of its development is that, firstly, it is necessary to define a range of parameters that reflect the stability (instability) of the company's activity. Secondly, it is necessary to combine parameters, which are divergent in their action and value; and, thirdly, harmoniously combine all the components of the financial stability, etc.

The methodology of the multidimensional economic and statistical analysis makes it possible to get the most accurate estimate, using the analysed blocks of parameters, comprehensively characterizing a company's activity, as of a specific date. Parameters are calculated for each block and the integrated parameter of the company's financial stability is calculated based on these parameters.

A need to define a form for the integrated parameter of the company's financial stability has become crucial. Most often, the stability parameters are calculated as the arithmetic mean of individual parameters. However, as to the meaning of the analysed integrated parameter, it corresponds more to the geometric mean, reflecting a relation between the parameters. Besides, the parameters under consideration help to show an actual situation at the company. The integrated parameter of the company's financial stability can be calculated using the following formula:

$I=\left(R_{c l} \cdot R_{a l} \cdot R_{e} \cdot R_{l} \cdot R_{w c} \cdot R_{a t} \cdot R_{a t}\right)^{1 / 7}$,

Where $R_{c l}$ is current liquidity ratio (short-term assets/ short-term liabilities); $R_{a l}$ is absolute liquidity ratio (financial resources and their equivalents/short-term liabilities); $R_{e}$ is equity ratio (capital/balance-sheet total); $R_{l}$ - leverage ratio [(short-term liabilities + long-term liabilities)/capital]; $R_{w c}$ - working capital to current assets ratio [short-term assets - short-term liabilities)/shortterm assets]; $R_{a t \text { - }}$ asset turnover ratio (sales revenues/ average annual stock value).

The integrated parameter formed in this way will help us to take into consideration the significance (contribution) of each component of the company's financial stability when performing the target function. A change in any of the included parameters leads to a change in the value of the integrated parameter of the company's financial stability.

An increase (decrease) in the integrated parameter of the company's financial stability in dynamics indicates an increase (decrease) in the level of stability and reliability of the company's activity, which is characterized by a positive/negative change in the increment function of the integrated parameter. Consequently, an increase in the parameter of the company's stability is associated with the positive increment of the integrated index in the base period $I(t+1)$ compared to the previous period $I(t): I(t+$ 1) $>I(t)$.

\subsection{Potential Financial Stability Function}

The basic method to study stepwise changes in the functioning of a system (from its stable to unstable condition) is to analyse if the potential function $F(x, I)$ has critical points, in which the derivative is 0 . The company's financial stability depends on a number of factors or control parameters, that is, the potential financial stability function can be presented as $F(x, I)$, where: $x$ is a control parameter. The parameter of company's potential function $x$, in its turn, is function $x=f\left(x_{1}, x_{2}, x_{3}, \ldots, x_{n}\right)$.

If the control parameter is positive, but shows a decreasing tendency, the system can be considered as close to a catastrophe. The catastrophe is a stepwise change of the system as a sudden system response to smooth changes in external conditions. If it has been found that relations between variables, which are illustrative for the system behaviour, can be described with certain equations, we can state that the system is instable and close to the catastrophe

Let us assume that the company is financially sound, it does not go bankrupt and gets some profit. The employees do their best to make the process go on. This happens 
with the certain integrated parameter $I$ and certain values of control parameters $x_{1}, x_{2}, x_{3}, \ldots, x_{n}$. Qualitatively, this situation is shown in Figure 2.
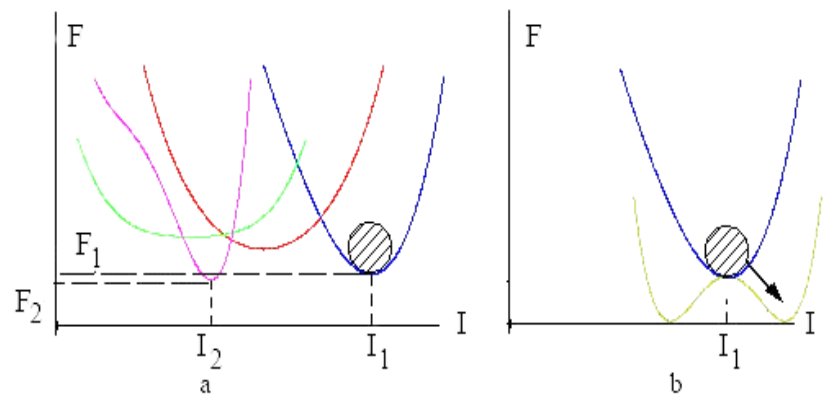

Figure 2. Company's financial characteristics. Source ${ }^{27}$.

In Figure 2, there are 4 graphs of potential function $F$ against index $I$. A company is conventionally designated with a crosshatched circle. The graphs have different sets of control parameters. When there is a smooth change in the control parameters, the graphs shift, their curves go steeper or flatter or become distorted. But all of them have something in common, i.e. the company's financial stability defined by one curve at least. This activity zone is called a pre-critical catastrophe zone.

Further on, the company undergoes the catastrophe, that is, goes bankrupt. Let us assume that the catastrophe occurs when $F$ function is zero. Geometrically, it comes from the fact that the previous, stable state, becomes unstable and the company falls down from it into another stable state, now with zero $F$ (Figure 2). In this case, with another set of control parameters, curve F (brown graph) has already two stable equilibrium states with zero function $F$ and one unstable equilibrium that separate them. This situation is called the overcritical catastrophe zone.

So, we can suggest that the catastrophe implies a change in quantity and quality of equilibrium states of a company. The set of parameters implying the catastrophe is called critical. When the company in its activities is close to this state, the catastrophe becomes more probable.

To improve the company's financial stability, one should know the exact relation of $f$, that is, know which value of the integrated parameter $I$ corresponds to changes in control parameters $x_{1}, x_{2}, x_{3}, \ldots, x_{n}$. Such a model is definitely hard to be developed. That is why we can use the catastrophe theory and get quite a simple model based on minimal knowledge of $f$ functions.

\subsection{Modelling Bankruptcy Probability following Catastrophe Theory}

The simple catastrophe theory is based on Thom's theorem and Arnold's classification. They defined simplest forms for stable and unstable relations in the systems ${ }^{12,15}$. Caspoid fold and cusp catastrophes are of the main interest in economic research. Using general mathematical reasoning, we are going to describe qualitatively the financial position of a company with changed $x$ parameters. We will make a research of the dependence on two or more parameters with other values considered noncritical. The selected parameters were used to build models of caspoid catastrophes approximating interrelations of changes in $x$ and $I$ :

fold catastrophes -

$F=I^{3}+x I$

cusp catastrophes -

$F=I^{4}+x_{1} I^{2}+x_{2} I$

\subsubsection{Fold Catastrophes}

The Equation (5) is a function, the nature of which depends on a value of parameter $x$. If this parameter is positive, the function is monotonic and its graph is a smooth and monotonically increasing function. But if parameter $x$ decreases, the type of the function changes at its zero value. At zero value of the parameter, the nature of a relation in the system changes, and this change is called bifurcation. If the parameter has a negative value, function $x$, described in the Equation (2), is a non-monotonic one. It has a maximum and a minimum ${ }^{27,22}$ at values $I= \pm x^{1 / 2}$.

The relation between variables in a neighbourhood of the origin of coordinates will be many-valued. One value for variable $F(x, I)$ will correspond to three differentvalues of the variable $I$. So, when the variable $F$ undergoes changes in a monotonic smooth way, the variable $I$ will change in a stepwise manner. That will be the catastrophe.

The functions that describe relations between variables, characterizing the behaviour of the economic system, can be obtained with econometric approaches. For instance, a relation between two variables can be shown in the Equation (7), the nature of which depends on a value of parameter $x$.

If the parameter $x$ is positive, the function is monotonic 
and its graph is a smooth, monotonically increasing curve. But if the parameter $\mathrm{x}$ decreases, the type of the function changes at its zero value. At zero, the nature of the relation in the system changes and this change is called bifurcation.

The relation between variables in a neighbourhood of the origin of coordinates will be many-valued. If it has been established that the relation between variables, characterizing the behaviour of the system, can be described by the Equation of the type (7), it can be said that the system demonstrates instability. If parameter $x_{1}$ is positive but there is a decreasing tendency, we can suggest that the system is close to the catastrophe. In both cases, the system should be further explored to find conditions and possible terms of the catastrophe and estimate its consequences. The graph of the fold catastrophe is presented in Figure 3.

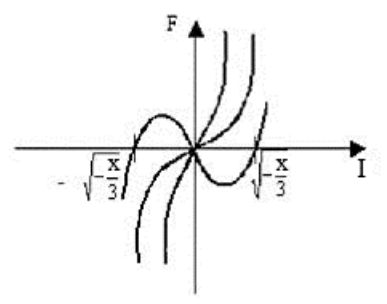

a

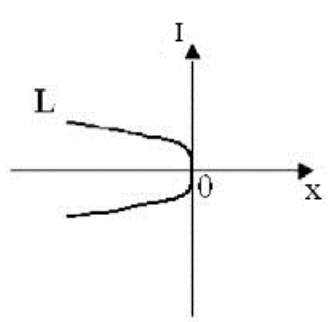

5
Figure 3. Geometry of the fold catastrophe. Source ${ }^{29}$.

At the point $(0,0)$, the function has a bend. When the function $F$ is perturbed by control parameter $x$, we get two possible states of the system.

- At $x>0$, the function has no critical points (the system is stable);

- At $x<0$, the function has two critical points (the system is unstable);

$I_{1}=-(-x)^{1 / 2}, I_{2}=(-x)^{1 / 2}$

Degenerate critical point $I=0$ of function $F$ splits into two non-degenerate ones under the influence of perturbation $x$ $<0$. Herein, there is instability of the fold catastrophe. Critical and degenerate points of this family can be found provided that the first order- and second order derivatives of function $F / I$ are equal to zero and form Equations:

$L=d F / d I=3 I^{2}+x=0$;

$\left.d^{2} F\right) / d I^{2}=6 I=0$.
The equilibrium curve $L$ (Figure 3 ) of the fold catastrophe looks like a great number of points $(x, I)$ on plane $F$, which satisfy the Equation (8). The upper sector of the parabola corresponds to local minimum points, and its lower sector to local maximum points of the function.

\subsubsection{The Cusp Catastrophe}

To interpret qualitatively the company's bankruptcy probability, the traditional cusp catastrophes are used (8). There are two variants of possible crises, corresponding to $I_{1}$ and $I_{2}$. The overall financial standing of a company is characterized by $I_{l}$, when the crisis develops gradually and $I_{2}$ corresponds to the situation when the crisis first develops gradually up to a tipping point and then, at the moment of the catastrophe, there is a severe decrease in possibilities for the stable performance of the company and there is a significant decline or an upturn in parameters' values, implying their deterioration.

Degenerate critical points of the family $I$ is found provided that the first order, second-order and thirdorder derivatives $I$ are equal to 0 , accordingly:

$d F / d I=4 I^{3}+2 x_{1} I+x_{2}=0 ;$

$d^{2} F / d I^{2}=12 I^{2}+2 x_{1}=0$

$d^{3} F / d I^{3}=24 I=0$.

The formula (12) can be used to find the cross-section of the cusp catastrophe in the plane $\left(x_{1}, I\right)$, which is a parabola Figures 4 and 5.

$x_{1}=-6 I^{2}$
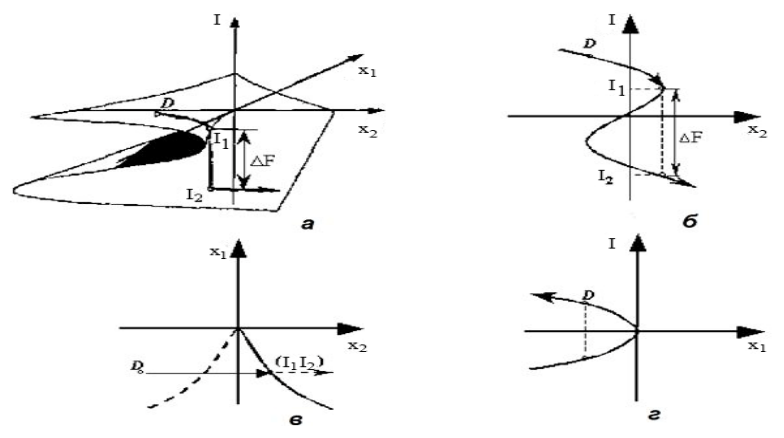

Figure 4. Geometry of cusp catastrophe (a and b). Source ${ }^{29}$. 

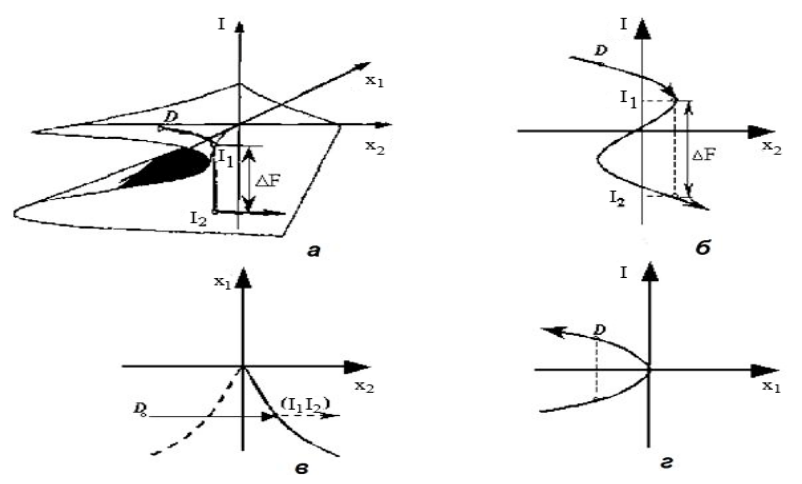

Figure 5. Geometry of cusp catastrophe (c and d). Source ${ }^{29}$.

Plugging (14) into Equation (11), we will get a crosssection of the cusp catastrophe in the plane $\left(x_{2^{\prime}} I\right)$, shown in Figure 5:

$x_{2}=8 I^{3}$.

Solving the system of equations (11) and (12) for $a$, we get the bifurcation set (Figure. 5):

$8 x_{1}^{3}+27 x_{2}^{2}=0$

The bifurcation set is a number of points of the plane, characterized by duality of their function. This set defines a zone of the system instability. When the system parameters (point D in Figure 4), while changing smoothly, cross the bifurcation set, the system passes from one stable equilibrium $I_{1}$ to another - $I_{2}$, in a stepwise manner.

Cusp catastrophes have one stable equilibrium state in the pre-critical zone (a pit in the potential function) and two stable and one unstable equilibrium in the overcritical zone (two pits separated by a peak):

Pre-critical and overcritical zones are defined by the following set of parameters.

Pre-critical zone:

$\mathrm{x}_{1}<0,-\infty>\mathrm{x}_{2}>\infty$

$\mathrm{x}_{1}>0,\left|\mathrm{x}_{2}\right|>2 / \mathrm{x}_{1}\left(1 / 3 \mathrm{x}_{1}\right)^{1 / 2}$

Overcritical zone:

$x_{1}>0,\left|x_{2}\right|<2 / 3 x_{1}\left(1 / 3 x_{1}\right)^{1 / 2}$

\section{Results and Discussion}

The canonical form of the cusp function is a particular case of the sustainable development index within the specially formed coordinate system. So, to build it, it is necessary to use the general non-linear function ${ }^{24,25}$. That is why as a function (8), we will analyze the fourth-degree polynomial function of the form:

$F=x_{1} I^{4}+x_{2} I^{3}+x_{3} I^{2}+x_{4} I$,

Where $x_{1}$ - active assets ; $x_{2}$ - liabilities; $x_{3}$-capital; $x_{4}$ - gross profits.

To find the general form for polynomial function $F_{\mathrm{o}}=$ $a_{0}+a_{1} I+a_{2} I^{2}+a_{3} I^{3}+a_{4} I^{4}$, we first found the dependence of function $F(x, I)$, using statistical data from 27 Kazakhstani companies, including those 7 , that had gone bankrupt and then approximating the findings got with the method of least squares, we received coefficients $\mathrm{a}_{\mathrm{i}}$ and defined $F_{o}$ dependence on the integrated parameter of the company's financial stability:

$F_{o}=2.81-2.79 I-0.12 I^{2}-0.87 I^{3}+1.80 I^{4}$

$F$-test $=0.53 ;$-test $=2.02$.

Based on the dependence (16), we have developed the logit-model to assess the bankruptcy probability, which looks like the following:

$P=1 /\left(1+e^{-F o}\right)$,

Where $e$ is base of the natural logarithm.

Managerial decisions following the model of the assessment for the company's bankruptcy probability are done in accordance with Table 2. It should be said that the assessment of the bankruptcy probability should be performed at certain intervals and the dynamics of its temporal change should be analysed. This will help managers to define more precisely the company's financial stability and take corresponding measures on time.

Table 2. Characteristics of company's bankruptcy probability following the author's model

\begin{tabular}{|c|c|}
\hline $\begin{array}{l}\text { Variation intervals of } \\
\text { bankruptcy probabilit }\end{array}$ & $\begin{array}{l}\text { Bankruptcy } \\
\text { probability }\end{array}$ \\
\hline $\mathrm{P}<0.7 \quad \mathrm{Lo}$ & ow probability \\
\hline $0.7<\mathrm{P}<0.8$ & Medium probability \\
\hline $\mathrm{P}>0.8$ & High probability \\
\hline
\end{tabular}

If $P$ value is less than 0.7 , the bankruptcy probability is low, if it is over 0.8 , the bankruptcy probability is high. 
Following the suggested method, we have performed the assessment of the bankruptcy probability for several Kazakhstani companies to give eventually some recommendations as how to avoid the bankruptcy Table 3. Financial statements of the companies were used as the informational background in the research.

The companies under consideration can be divided into three groups. Companies from the first group are characterized by the high rate of sales revenue growth and the low risk level. In this case, it is allowed for the company's managers to avoid adventurous projects aimed at the high profit, playing safe instead. Consequently, their options for strategic actions should be the following: Avoidance of unreliable partners and risk projects, insurance for economic risks and a search for guarantors.

Companies from the second group are characterized with the average business risk and average market growth rate of sales revenue. This means that despite being enough venturous, the business is successfully developing. The following situation requires managerial decisions of risk distribution. The companies should diversify their risk by means of activities on diversifying types of activities, supply, sale and investments, as well as distributed responsibility between participants and temporal distribution for operations.

Companies from the third group have the high risk rate and below-average market growth rate of revenue. In this case, a company's management should make a decision to apply a method of risk localizatione29,30. That is, it is necessary to find out bottlenecks in their activity and as far as possible, eliminate them from the company or establish responsibility centres ${ }^{3}$. Having defined the most dangerous zone in the activity, the company can bring it under control and thus reduce the bankruptcy level of the company.

For a further comparison with the proposed model, the assessment of the bankruptcy probability for the considered companies was also performed according to Altman's Z-score model and Savitskaia's Z-model (no Kazakstani models were used). The findings are given in Table 4.

Table 3. Assessment of companies' bankruptcy probability following the suggested model

\begin{tabular}{lccl}
\hline Company's name & $\mathrm{F}_{\mathrm{o}}$ function value & $\mathrm{P}$ criterion value & Bankruptcy probability \\
\hline KazMunayGas, JSC & 0.63 & 0.65 & Low probability \\
Kaztemirtrans, JSC & 0.77 & 0.68 & \\
AirportPavlodar, JSC & 0.77 & 0.68 & \\
Kazakhtelecom, JSC & 0.92 & 0.71 & Medium probability \\
Transtelecom, JSC & 1.14 & 0.76 & \\
KazAgroProduct, JSC & 1.28 & 0.78 & \\
KazTransOil, JSC & 2.11 & 0.89 & High probability \\
Kazteleradio, JSC & 2.39 & 0.91 & \\
Passenger Transportation, JSC & 4.23 & 0.99 & \\
\hline
\end{tabular}

Table 4. Assessment of the companies' bankruptcy probability following Z-Score and Z models

\begin{tabular}{lcl}
\hline Company's name & $\begin{array}{c}\text { Z-Score value and } \\
\text { bankruptcy probability }\end{array}$ & $\begin{array}{l}\text { Z value and bankruptcy } \\
\text { probability }\end{array}$ \\
\hline KazMunayGas, JSC & 2.51 Medium & 6.56 Low \\
Kaztemirtrans, JSC & 0.96 Very high & $2.88 \mathrm{High}$ \\
AirportPavlodar, JSC & 2.88 Not high & $9.10 \mathrm{NA}$ \\
Kazakhtelecom, JSC & 2.96 Not high & $4.49 \mathrm{Medium}$ \\
Transtelecom, JSC & 0.95 Very high & $-1.66 \mathrm{Maximum}$ \\
KazAgroProduct, JSC & 3.04 Negligible & $9.94 \mathrm{NA}$ \\
KazTransOil, JSC & 3.46 Negligible & $2.95 \mathrm{High}$ \\
Kazteleradio, JSC & 7.05 Negligible & 8.79 NA \\
Passenger Transportation, JSC & 1.40 Very high & $1.72 \mathrm{High}$ \\
\hline
\end{tabular}


According to Altman's Z-Score (1), such companies, as Kaztemirtrans, JSC, Transtelecom, JSC and Passenger Transportation, JSC are in the zone of the highly probable bankruptcy $(Z<1.81)$; KazMunayGas, JSC is in the zone of the medium probability of bankruptcy $(1.81<\mathrm{Z}<$ 2.675). According to our model, Kaztemirtrans, JSC is in the zone of the low probability of bankruptcy, while Transtelecom, JSC is in the zone of the medium probability of bankruptcy and KazMunayGas, JSC is in the zone of the low probability of bankruptcy. The forecast results coincide for Passenger Transportation, JSC.

Differences in assessment results are due to the above-mentioned causes. That is, overseas models are trustworthy but almost not applicable in Kazakhstan conditions due to the differences in companies' business and regulatory framework

\section{Conclusion}

The problem of assessment regarding the companies' financial position and in particular, forcasting the probability of their bankruptcy has remained one of the most important in the modern economy of Kazakhstan. So far, there have been developed a number of methods to assess and forcast bankruptcy, but every method has its weak and strong points. In this aspect, the suggested method to assess the company's bankruptcy probability based on the catastrophe theory is a new approach to solving the considered problem and it has certain theoretical and practical significance.

Its theoretical significance lies in the fact that the application of the catastrophe theory elements allows for a more accurate assessment of a possible change in the company' stable development, influenced by determinant criteria and also helps to identify possible changes in the company's economic situation, when characteristic criteria change. The practical significance of this approach lies in its ability to predict on time a developing mismatch in a company's structure, define the moment of entering a critical zone. It serves as a signal for development and implementation of measures to affect the company preventing a fall in its developmental potential.

\section{References}

1. Altman E, Hotchkiss E. Corporate financial distress and bankruptcy: Predict and avoid bankruptcy, analyse and in- vest in distressed debt. 3rd ed. John Wiley and Sons, Limited; 2006.

2. Beaver W. Financial ratios as predictors of failure. Empirical research in accounting selected studies. Journal of Accounting Research (Suppl). 1966; 4:71-111.

3. Forecasting Bankruptcy Probability by Lis's Model. 2016. Available from: www.anfin.ru/prognozirovanie-bankrotstva-po-modeli-lisa/

4. Taffler's Model. 2016. Available from: www.1-fin. $\mathrm{ru} /$ ? id $=281 \& \mathrm{t}=978 \& \mathrm{str}$

5. Conan J, Holder M. Explicatives variables of performance and management control. [PhD Thesis]. CERG: Universite Paris Dauphine; 1979.

6. Fulmer J, Moon J, Gavin T, Erwin M. A bankruptcy classification model for small companies. Journal of Commercial Bank Lending; 1984.

7. Springate GLV. Predicting the possibility of failure in a Canadian Company. [Unpublished MBA Research Project]. Simon Fraser University; 1978.

8. Sheremet AD, Negashev EV. Technique of financial analysis for commercial organizations activities. Moscow: INFRA-M; 2008.

9. Saiffulin and Kadykov's Models. 2016. Available from: www.studopedia.org/10-73970.html

10. Kovalev VV. Financial analysis: Methods and procedures. Moscow: The Finance and Statistics Publishing; 2011.

11. Savitskaia GV. Analysis of corporate business activities. Moscow: SIC INFRA-M; 2016.

12. Nedosekin AO. Methodological bases for modelling financial performance using fuzzy multiple descriptions. [Ph.D (Econ) Thesis]. Saint Petersburg; 2003.

13. Thom R. Structural stability and morphogenesis. Moscow: Logos; 2002.

14. Arnold VI. Catastrophe theory. Moscow: Science; 1990.

15. Poston T, Stewart I. Catastrophe theory and its applications. Moscow: Peace; 1980.

16. Thompson JMT. Instabilities and catastrophes in science and engineering. Moscow: Peace; 1985.

17. Gilmore R. Applied catastrophe theory. Moscow: Peace; 1984.

18. Zang VB. Synergetic economics. Time and Change in Nonlinear Economic Theory. Moscow: Peace; 1999.

19. Pchelenok NV, Maslov BG. Foreign and Russian methods of bankruptcy prediction. Financial Accounting. 2005; 5:ID24167.

20. Kuznetsov AP. Fluctuations, catastrophe, bifurcation, chaos. Saratov: Izd GosUNTs College; 2000.

21. Nedeljko NS. Using catastrophe theory to analyse economic systems' behaviour. Vestnik of MSTU. 2010; 13(1):223-7.

22. Asaul MA. Ensuring stability of enterprises in investment and construction. [PhD (Econ.) Thesis Abstract]. Saint Petersburg; 2008.

23. Oleynik YT. Microdynamics in innovative economic systems. Methods of Discreet Peculiarities in Problems of Mathematical Physics (DIPED-2009). Proceedings of the XIV International Seminar by V. N. Karazin Kharkov National University; Kharkov, Ukraine. 2009; 2-X: 390-3. 
24. Kovalenko AV, Urtenov MK, Trakhova SS. Mathematic modelling of financial and economic crisis at an enterprise with used canonical catastrophes of fold and cusp. Scientific Journal of KubSAU. 2010; 63:1-15.

25. Zenchenko SV, Egorkin EA. Catastrophe theory applied in stability assessment at credit organization. Herald of the North Caucasus Humanitarian and Technical Institute. 2014; 19:22-6.

26. Egorkin EA. Improving methods to assess financial stability at Commercial Bank. [Cand Sci (Econ.) Thesis Abstract]. Makhachkala; 2015.

27. Bushuyev AB. Mathematical modelling for technical creativity processes. Saint Petersburg: Saint Petersburg State ITMO University; 2011.
28. Alekseev YuK. Introduction to catastrophe theory. Moscow: MSU; 2000.

29. Stewart I. Catastroph secrets. Moscow: Peace; 1987.

30. Kuzmin EA. Fundamentals in systematics of uncertainty management theory. Mediterranean Journal of Social Sciences. 2015; 6(5S2):380-9.

31. Taklif A, Shokouhian R, Arasteh AS, Dalfard VM. Quantifying risk analysis using database in industrial investment projects by Topsis method. Indian Journal of Science and Technology. 2011; 4(7):1-10.

32. Tumenbayeva OS, Zhaksybekova GN. Implementation of the integrated system of risk management in the Banks of Kazakhstan. Indian Journal of Science and Technology. 2016; 9(5):1-8. 